\title{
Strategies of coping with stress and the sport results of alpine skiers and tennis players
}

\author{
Michał Knittel ${ }^{1}$, Monika Guszkowska² \\ ${ }^{1}$ Faculty of Physical Education, Józef Piłsudski University of Physical Education, Warsaw, Poland; ${ }^{2}$ Faculty of Tourism \\ and Recreation, Józef Piłsudski University of Physical Education, Warsaw, Poland
}

\section{Summary}

Study aim: The aim of the study was to determine the relationship between strategies of coping with stress caused by participating in sport competitions and the results of those competitions, as well as to differentiate the applied coping strategies according to the practiced sport discipline (tennis and alpine skiing).

Material and methods: The study encompassed 83 university students (39 women and 44 men) who were participating in the Polish University Championships in tennis $(\mathrm{n}=32)$ and alpine skiing $(\mathrm{n}=51)$. The research was based on the Coping Inventory for Competitive Sports (CICS) questionnaire and was adapted to Polish conditions by the authors of this study. The results achieved during the competition were also recorded during the study.

Results: Possibly due to the nature of their sport, the tennis players used coping actions to a greater extent than the alpine skiers, regardless of the nature of the action. The application of task-focused strategies and the lower use of emotion-focused and avoidance strategies allowed the athletes to achieve higher results during the competition.

Conclusions: Task-focused strategies should be developed in order to improve the competitive results of athletes. Taking the situational nature of the coping strategies into consideration, coping strategies can be designed and modified to improve the achieved results.

Key words: Stress - Coping - Coping strategies - Alpine skiing - Tennis

\section{Introduction}

The issue of coping with stress in sport has been studied by researchers all over the world $[2,3,5,16]$. The improvement of competition results requires the athlete to have better mental preparation, including a better ability to cope with stress caused by participating in a sport competition.

Athletes adopt various individual approaches to dealing with the stress caused by taking part in a competition. The coping actions undertaken in particular competitive circumstances are called coping strategies. Kaiseler et al. [13] distinguished the following coping strategies used by athletes from various disciplines: 1) problem-focused strategies (active coping, planning and increasing effort); 2) emotion-focused strategies (seeking emotional support, humour and wishful thinking); and 3) avoidance strategies (denial and disengagement). However, this classification is universal and does not only concern situations in sport, where the approach adopted for measuring the coping strategies is important.

Researchers have pointed out the necessity of using inventories or scales that are specific for sport [4], in which the contents of the items refer specifically to situations related to sport competitions and/or training. The Coping Inventory for Competitive Sports (CICS) is an example of such a tool. Its authors have distinguished 10 coping strategies which are classified into three groups: 1) task-oriented strategies (mental imagery, effort expenditure, thought control, seeking support, relaxation and logical analysis); 2) distraction-oriented strategies (distancing and mental distraction); and 3) disengagement-oriented strategies (venting of unpleasant emotions and resignation) [7].

It can be assumed that the efficiency of the above strategies will be diversified according to the athletes' mental traits and to the specific sport discipline. Therefore, it is justified to compare the coping strategies preferred in different sports, both with regard to their frequency of use and to their relationship with the outcomes in competitions. 
This seems to be particularly justified in individual sports, where the responsibility for the result rests with just one person. The many variables that influence the results in team sports make it more difficult to find a relationship between coping strategies and sport results.

Furthermore, individual disciplines differ in terms of the nature of the stress that occurs during a competition. In some disciplines, such as alpine skiing, pre-competition stress plays a significant role. Once the rivalry starts, which usually lasts for just a short period of time (a few minutes at most), the activation related to stress decreases. However, disciplines such as tennis require a sportsperson to cope with stress not only before a competition, but also during a match. Each point, the number of which frequently amounts to several hundred within a few hours, causes tension and requires the athlete's focus [9]. Because the disciplines selected for this study, i.e. tennis and alpine skiing, differ in terms of the mental resilience required from the athletes, comparing them with regard to the strategies of coping with stress caused by participating in a competition seems justified.

Studies by Pensgaard and Roberts [19] offer examples of stress research in the representatives of winter sports. The authors evaluated the athletes' coping strategies using the COPE questionnaire, where the strategies were divided into problem-focused strategies and emotion-focused strategies. According to the authors, it was difficult to determine whether a strategy was effective, and if the preferences for coping remained the same regardless of the time and situation. This difficulty was caused, at least partially, by the fact that the competition results were not registered in the study. The research encompassed the Norwegian representatives at the Winter Olympic Games; however, as the study did not differentiate between disciplines, no conclusions about alpine skiers in particular can be made on its basis.

Many studies indicate that the majority of tennis players increase their focus and prevent the intensification of stress by performing various match rituals, such as repeating stereotypical behaviours numerous times (bouncing a tennis ball a certain number of times before a serve, adjusting the strings in the racket or adjusting their attire, walking with a certain rhythm, running up to a chair, throwing their opponent a look, clenching their fists and smiling at the audience, etc.) [14]. The aim of the abovementioned activities is not to minimise stress, but to increase the athlete's ability to handle it [9]. A complete reduction of excitation is impossible, and is even inadvisable. What is important is the athlete's ability to transform this tension into a positive excitation and to cope with negative emotions.

The techniques for handling anxiety used by tennis players, as distinguished by experts [18], include: focusing on a specific item, sensory sensitivity, positive memories and tuning out certain thoughts. The author points out the necessity of tuning out certain thoughts. After a mistake, before an important play or after a wrong decision made by a referee, negative thoughts will often appear in the mind of a tennis player. The irritated player will make another mistake during the subsequent play, and may become even more irritated. This causes a phenomenon referred to as 'the vicious cycle'.

On a micro level (a single match), a tennis player's optimal mental functioning is a matter of developing autoregulation and the ability to control thoughts, emotions and behaviours during a play and during the breaks in a match [10]. This is often particularly visible during the 'dead moments' of a match. These moments should be filled with particular routine actions, breathing actions and body language [11]. Before each point, a tennis player can prepare themselves for it in an individual way; for example, by performing pre-serve mental exercises. These exercises generally involve repeating in one's mind the sense, tactics, technique and effectiveness of a serve before serving [15].

However, researchers have fairly seldom verified the efficiency of coping strategies proposed by psychologists, and examined whether the strategies preferred by athletes vary according to the sports discipline. The aim of this study was to determine the relationship between strategies of coping with stress caused by participating in competitions and the results of those competitions, as well as to differentiate between the applied strategies according to the practiced sport (tennis and alpine skiing).

\section{Material and methods}

The study encompassed 83 university students (39 women and 44 men) who were participating in the Polish University Championships in tennis $(\mathrm{n}=32)$ and alpine skiing $(\mathrm{n}=51)$.

The Coping Inventory for Competitive Sports (CICS) was used, which enables researchers to investigate the strategies, that is, the actions undertaken before and during a particular sports competition. The original French version of the research tool comprises 39 items and allows for the measurement of 10 strategies: mental imagery, effort expenditure, thought control, seeking support, relaxation, logical analysis, distancing, mental distraction, venting of unpleasant emotions and resignation [12].

For the present study, this tool was adapted to Polish conditions. The Polish version of the CICS questionnaire comprises 36 items grouped into 10 scales that are consistent with the original version. The reliability of the questionnaire, as expressed with Cronbach's alpha, equals 0.86 ; and the reliability of each scale ranges from 0.63 to 0.89 . Furthermore, the validity of the tool was confirmed 
by correlating the results from each scale with the results of Endler and Parker's Coping Inventory for Stressful Situations (CISS). The participants completed the questionnaire immediately after finishing their match (tennis players) or descent (skiers), and described the measures they had applied before and during the rivalry.

Furthermore, the results of the competition were registered and were treated as the indicator for the efficiency of coping strategies used in the given competitive circumstance. For the purpose of the statistical analyses, the results obtained by the study participants were referred to as the class of the results, and were classified into three categories: low, medium and high. The respondents who obtained the low class of results placed the lowest in the competition; those in the medium class ranked in the middle; and those placed in the high class took part in the finals of the competition.

A two-way analysis of variance (the class of the results $\times$ discipline) from the SPSS Statistics software (Version 22) was used to determine the differences according to the class of the results and the sport discipline. In case of the differentiation according to the class of the results, the Bonferroni post-hoc test was used. Additionally, the Pearson product-moment correlation coefficients between coping strategies and the class of the results were calculated.

\section{Results}

The results of the analysis of variance are presented in Table 1. The tennis players displayed a stronger tendency to use the following strategies: seeking support, logical analysis, distancing, mental distraction, venting of unpleasant emotions and resignation. The results of the skiers' use of the strategies were lower than those of the tennis players in all cases.

The greatest diversification of coping strategies, according to the class of the results, was observed in the case of the 'resignation' and 'mental distraction' strategies. Both of these strategies were used most frequently by the athletes who achieved the lowest results. The same relationship can be observed in the case of "venting of unpleasant emotions' and (at the level of a tendency) 'distancing'. The Bonferroni tests for the above-mentioned variables indicated a diversification between the low class and the high class of the results at the significance level $p<0.01$.

On the other hand, the task-focused strategies were used more often by the athletes who achieved better result. In the case of 'thought control', the diversification was statistically significant; and in the case of 'effort expenditure',

Table 1. Strategies of coping with stress in sport according to the practiced sport discipline and the class of the results

\begin{tabular}{|c|c|c|c|c|c|c|c|c|c|c|c|}
\hline \multirow{3}{*}{ Variable } & \multicolumn{3}{|c|}{ Class of result } & \multicolumn{2}{|c|}{ Discipline } & \multicolumn{6}{|c|}{ ANOVA } \\
\hline & \multirow{2}{*}{ Low } & \multirow{2}{*}{ Medium } & \multirow{2}{*}{ High } & \multirow{2}{*}{ Tennis } & \multirow{2}{*}{ Skiing } & \multicolumn{2}{|c|}{ Discipline } & \multicolumn{2}{|c|}{ Class of result } & \multicolumn{2}{|c|}{ Interaction } \\
\hline & & & & & & $F$ & $p$ & $F$ & $p$ & $F$ & $p$ \\
\hline $\begin{array}{l}\text { Mental } \\
\text { imagery }\end{array}$ & $12.08 \pm 2.72$ & $11.51 \pm 3.46$ & $12.95 \pm 3.59$ & $13.00 \pm 2.63$ & $12.09 \pm 3.65$ & 1.022 & 0.314 & 1.412 & 0.248 & 2.420 & 0.093 \\
\hline $\begin{array}{l}\text { Effort } \\
\text { expenditure }\end{array}$ & $9.88 \pm 2.59$ & $10.53 \pm 2.66$ & $11.10 \pm 2.50$ & $11.19 \pm 2.57$ & $10.52 \pm 2.59$ & 1.638 & 0.203 & $2.504^{\mathrm{a}}$ & 0.086 & 2.298 & 0.105 \\
\hline $\begin{array}{l}\text { Thought } \\
\text { control }\end{array}$ & $9.50 \pm 2.74$ & $10.84 \pm 2.39$ & $11.14 \pm 2.68$ & $10.63 \pm 2.54$ & $10.78 \pm 2.69$ & 0.206 & 0.651 & $4.531^{b}$ & 0.013 & 1.129 & 0.327 \\
\hline $\begin{array}{l}\text { Seeking } \\
\text { support }\end{array}$ & $9.79 \pm 4.18$ & $10.65 \pm 4.01$ & $9.41 \pm 3.98$ & $11.53 \pm 4.17$ & $9.36 \pm 3.86$ & 6.711 & 0.011 & 1.313 & 0.273 & 0.136 & 0.873 \\
\hline Relaxation & $12.13 \pm 3.38$ & $10.63 \pm 3.51$ & $12.13 \pm 3.97$ & $11.75 \pm 3.83$ & $11.59 \pm 3.76$ & 0.204 & 0.653 & 1.173 & 0.313 & 3.587 & 0.031 \\
\hline $\begin{array}{l}\text { Logical } \\
\text { analysis }\end{array}$ & $8.04 \pm 2.65$ & $8.02 \pm 2.69$ & $7.71 \pm 3.06$ & $10.16 \pm 1.94$ & $7.13 \pm 2.70$ & 28.920 & $<0.001$ & 0.058 & 0.943 & 0.204 & 0.816 \\
\hline Distancing & $7.67 \pm 2.41$ & $6.19 \pm 2.79$ & $5.90 \pm 2.80$ & $8.34 \pm 2.91$ & $5.66 \pm 2.42$ & 22.594 & $<0.001$ & 2.641 & 0.075 & 0.095 & 0.910 \\
\hline $\begin{array}{l}\text { Mental } \\
\text { distraction }\end{array}$ & $10.17 \pm 3.73$ & $8.33 \pm 3.50$ & $7.37 \pm 2.84$ & $10.53 \pm 3.91$ & $7.44 \pm 2.81$ & 26.253 & $<0.001$ & 8.168 & $<0.001$ & 2.151 & 0.121 \\
\hline $\begin{array}{l}\text { Venting of } \\
\text { unpleasant } \\
\text { emotions }\end{array}$ & $10.71 \pm 5.35$ & $9.37 \pm 4.51$ & $8.11 \pm 4.49$ & $14.31 \pm 4.42$ & $7.28 \pm 3.36$ & 91.960 & $<0.001$ & 3.385 & 0.037 & 2.463 & 0.089 \\
\hline Resignation & $11.88 \pm 4.28$ & $9.98 \pm 3.46$ & $7.83 \pm 2.84$ & $11.16 \pm 4.29$ & $8.67 \pm 3.24$ & 17.004 & $<0.001$ & 16.675 & $<0.001$ & 5.745 & 0.004 \\
\hline
\end{tabular}

$\mathrm{a}$ - the results at the level of a tendency are italicised; $\mathrm{b}$ - the statistically significant results are in bold 
the diversification reached the level of a tendency. In the case of thought control, visible differences occurred between the worst and the best sportspersons.

As far as the 'relaxation' strategy is concerned, there was a significant correlation between the class of the results and the sport discipline. For 'mental imagery', this correlation reached the level of a tendency. Among the athletes with the low results, the tennis players used these two strategies less frequently than the skiers; and in the two other classes of results, the tennis players used these strategies more often than the skiers (Fig. 1 and Fig. 2).

In the case of 'venting of unpleasant emotions', the correlation reached the level of a tendency, and the tennis players from all three classes of results used this strategy more frequently than the skiers (Fig. 3).
The main reason for the diversification of the 'resignation' strategy was its frequent use by the tennis players who achieved the low class of results (Fig. 4).

The investigation of the linear correlations between the coping strategies and the class of the results (regardless of the practiced sport) revealed significant positive correlations between the task-focused strategies and the class of the results; while the emotion-focused strategies and avoidance strategies correlated negatively with the class of the results. The relevant data are presented in Table 2 . The more that the 'effort expenditure' was increased and the higher the levels of 'thought control', the better the athlete's results were. In turn, the higher results correlated with a lower level of such strategies as 'distancing', 'mental distraction', 'venting of unpleasant emotions' and

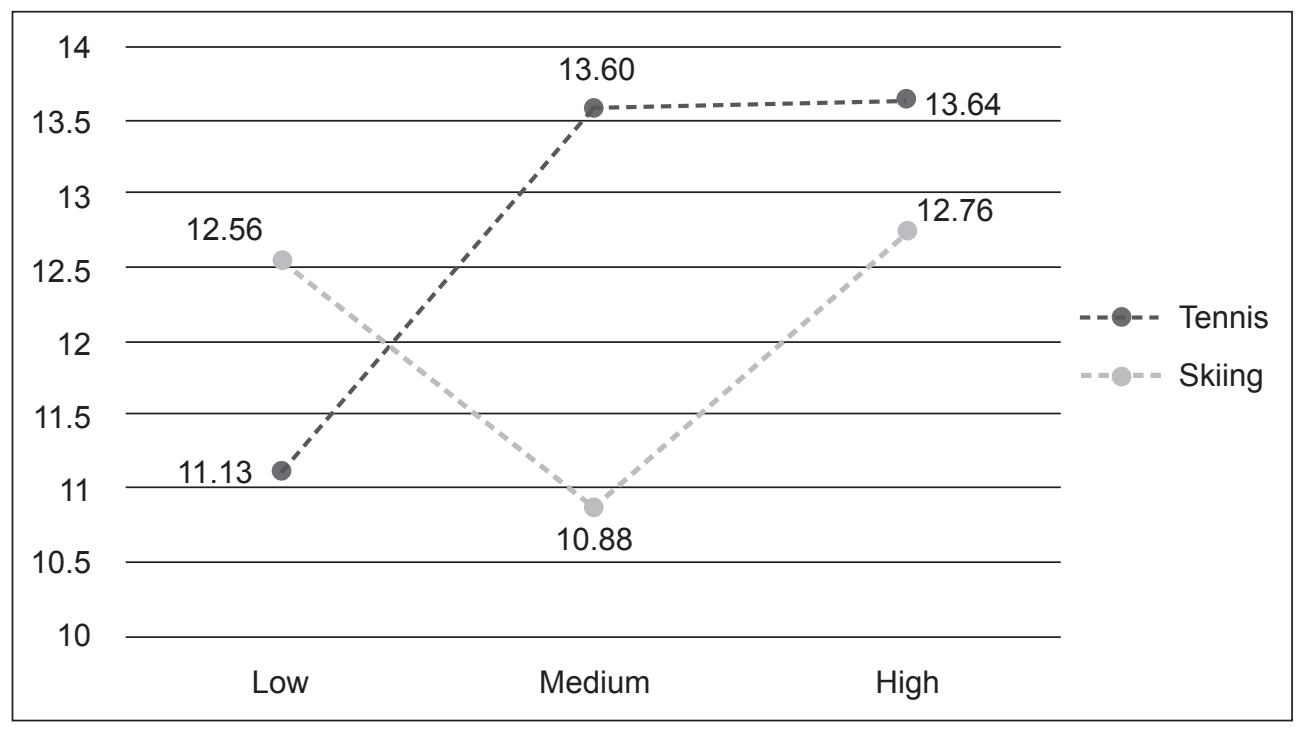

Fig. 1. 'Mental imagery' according to the practiced discipline and the class of the results

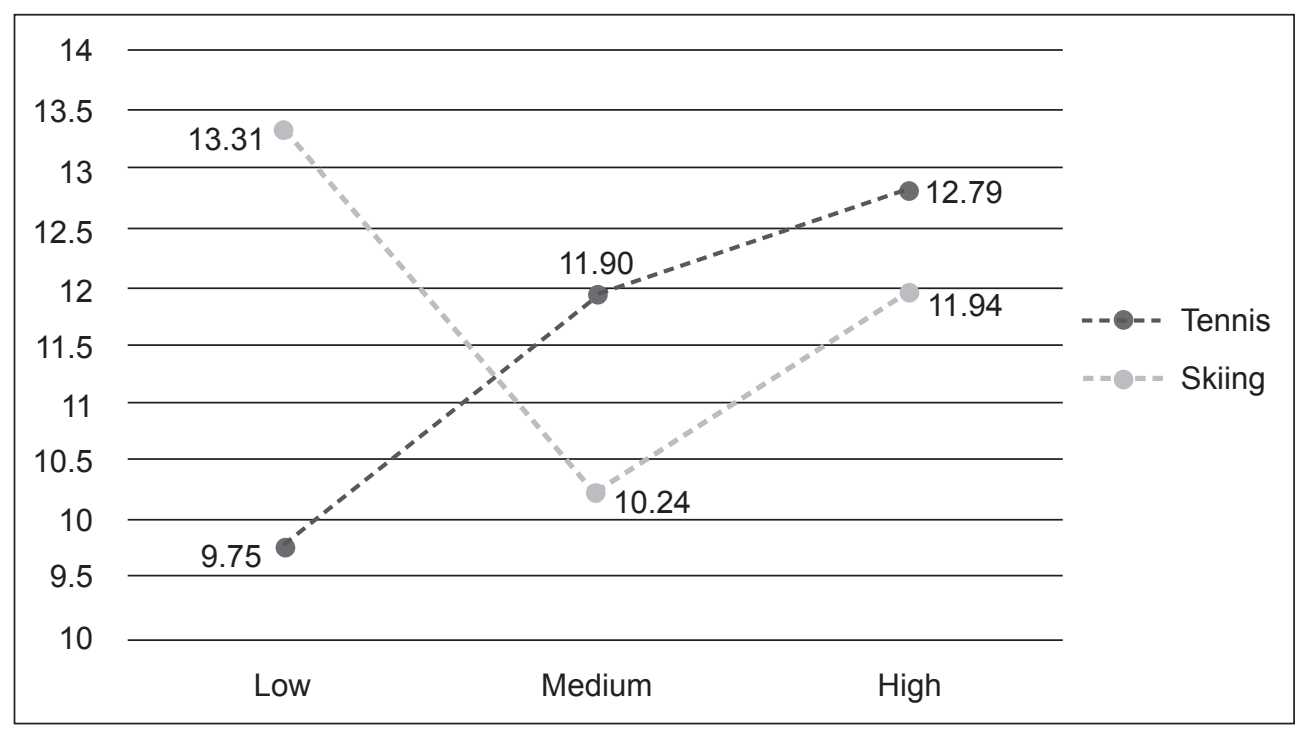

Fig. 2. 'Relaxation' according to the practiced discipline and the class of the results 


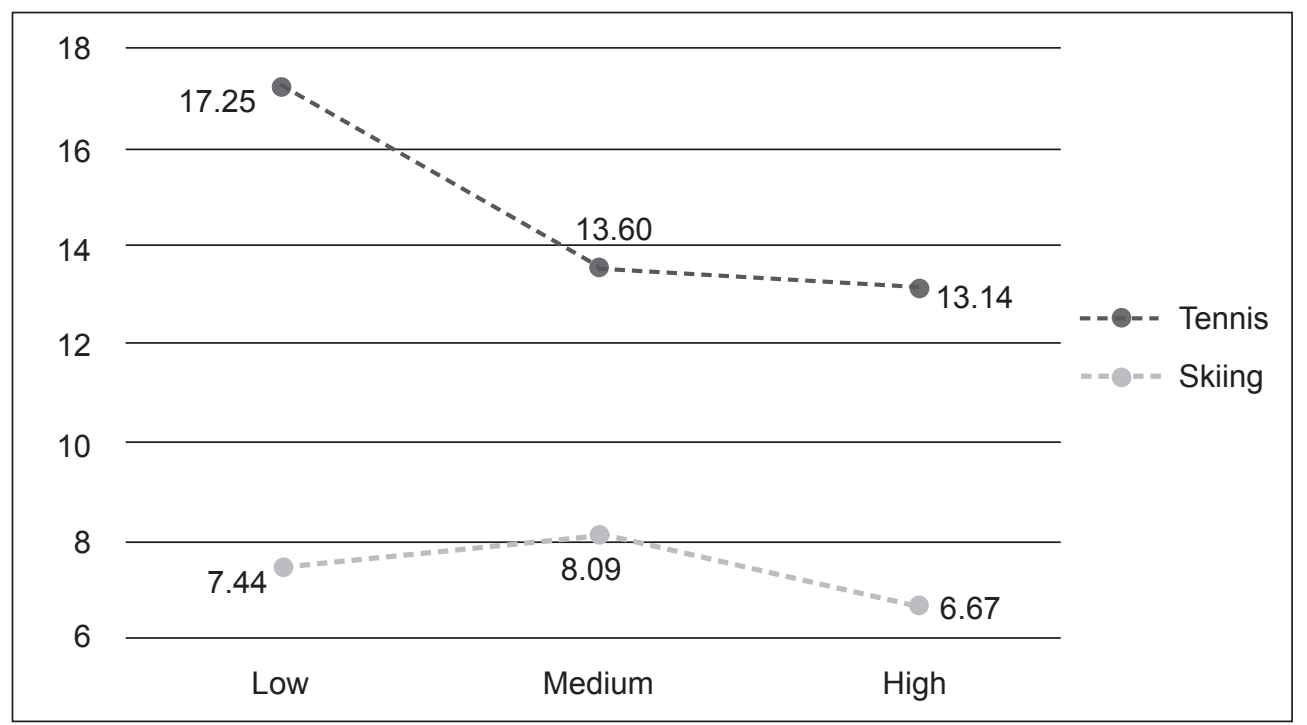

Fig. 3. 'Venting of unpleasant emotions' according to the practiced discipline and the class of the results

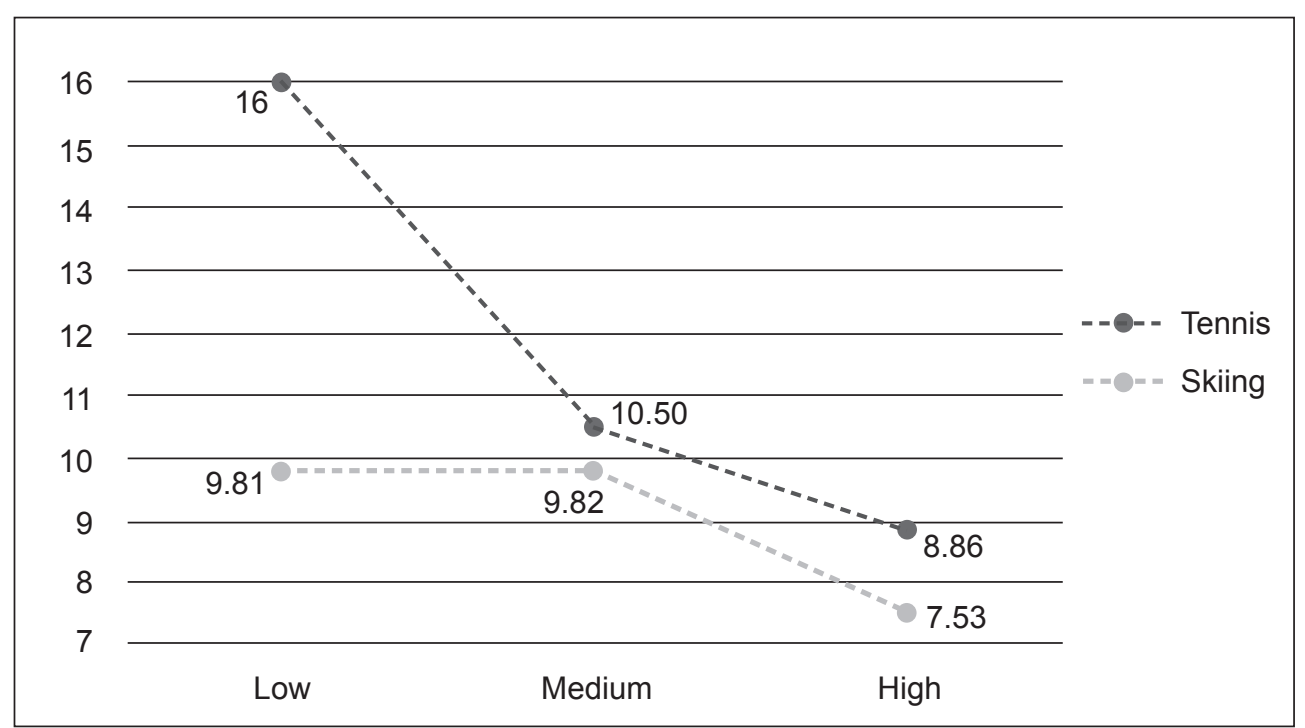

Fig. 4. 'Resignation' according to the practiced discipline and the class of the results

'resignation'. The strongest such correlation was observed for the 'resignation' strategy.

\section{Discussion}

The study revealed a diversification in coping strategies according to the sport discipline and class of the results. Tennis players were characterised by a stronger tendency to use the following strategies: seeking support, logical analysis, distancing, mental distraction, venting of unpleasant emotions and resignation. These results supplement other reports indicating coping strategies in tennis [9]. The fact that many of the above-mentioned strategies are used more often by tennis players results in the conclusion that this stems from the nature of this sport. For example, it seems justified that 'logical analysis' is the domain of tennis players, who have to keep continuously analysing the situation on the tennis court and keep adjusting their tactics to adapt to their opponents' actions. 'Distancing' is another example. Tennis requires the players to quickly forget about an unsuccessful play or a lost point, and to focus on further successful actions. The same can be said about 'mental distraction' (diverting attention). The purpose of the match rituals used by tennis players is to divert their attention from potential stressors and to help control excitation. 'Seeking support' may involve talking to one's coach during a break, a practice that has been 
Table 2. Relationships between coping strategies and the class of the results

\begin{tabular}{lcc}
\hline \multirow{2}{*}{ Strategy } & \multicolumn{2}{c}{ Class of result } \\
\cline { 2 - 3 } & $r$ & $p$ \\
\hline Mental imagery & 0.135 & 0.126 \\
Effort expenditure & $\mathbf{0 . 1 7 7 ^ { \mathbf { a } }}$ & $\mathbf{0 . 0 4 3}$ \\
Thought control & $\mathbf{0 . 2 1 2}$ & $\mathbf{0 . 0 1 6}$ \\
Relaxation & 0.052 & 0.554 \\
Logical analysis & -0.051 & 0.568 \\
Seeking support & -0.070 & 0.429 \\
Distancing & $\mathbf{- 0 . 2 1 3}$ & $\mathbf{0 . 0 1 5}$ \\
Mental distraction & $\mathbf{- 0 . 3 0 0}$ & $\mathbf{0 . 0 0 1}$ \\
Venting of unpleasant emotions & $\mathbf{- 0 . 2 0 9}$ & $\mathbf{0 . 0 1 7}$ \\
Resignation & $\mathbf{- 0 . 4 2 6}$ & $<\mathbf{0 . 0 0 1}$ \\
\hline
\end{tabular}

$\mathrm{a}$ - the statistically significant results are in bold

allowed in women's matches for several seasons. Additionally, tennis players often use 'mental imagery' in order to visualise a successful action [15].

'Relaxation' was often used by both tennis players and skiers. In both disciplines, breathing exercises and exercises to relax the muscles were performed [11]. The skiers generally try to remove the tension that exists in pre-competitive circumstances, after which the short descent takes place. However, the tennis players have to undertake such actions both before their match and during the competition. It should be mentioned that alpine skiers should not focus too much on 'relaxation', which may result in a low outcome.

In light of the obtained results, it seems justified to conclude that those tennis players that used coping strategies aimed at relieving tension, such as 'venting of unpleasant emotions' and 'resignation', were usually considered to be less effective.

The strategies involving 'resignation', 'mental distraction' and 'venting of unpleasant emotions' were used the most often by the athletes who achieved the lower results. However, in the case of 'thought control', s reverse relationship was observed, i.e. the strategy was most popular among the sportspersons with high results. The relationships between 'relaxation' and the results, and 'resignation' and the results, differed between the two disciplines. Tennis players with low results used 'relaxation' less frequently than the skiers with low results; while the tennis players who achieved a medium and high class of results used 'relaxation' more often. It was also observed that the tennis players who achieved low results often used the strategy of 'resignation'.

Furthermore, the study determined that the strategies of 'effort expenditure' and 'thought control' correlated positively with the class of the results. Higher results were accompanied by low levels of emotion-focused and avoidance strategies. This relationship has been confirmed in many studies $[6,8,17,20]$. Furthermore, Aldwin [1] believes that problem-focused strategies are more adaptational than emotion-focused and avoidance strategies. Other studies indicate mental imagery training to be an effective coping action [21]. Therefore, it can be assumed that this strategy would be effective both in tennis and in alpine skiing. Before starting, the skiers visualise their descent and recall the course with their eyes closed. In turn, some tennis players do this before each point. This shows that task-focused strategies are often used by the most highly-ranked sportspersons.

The results of this research indicate that coping strategies undertaken during competitions are of high importance. This is proven by the strong and numerous correlations between coping strategies and the results. Taking the situational nature of such actions into account, they may be designed and modified to improve the athletes' effectiveness in a competition.

The possibilities for generalising the results of this study are limited. The study encompassed sportspersons performing at diversified levels. Many of the participants often represent Poland at international events; however, an athlete's licence is not required for participating in the Polish University Championships, which makes the championships an amateur event. At this stage, generalising the conclusions over populations that differ from the one chosen for the study group is unjustified. The revealed relationships should therefore be verified in further studies encompassing events that bring together athletes from many disciplines, and of an increasingly higher level of sport.

This study was based on the Polish adaptation of the CICS questionnaire. This research tool is characterised by satisfactory psychometric features and may be used in further studies on the coping strategies undertaken by Polish sportspersons. Additionally, conducting a study with a higher number of participants from various sport disciplines would allow for the normalisation of the tool.

The results of the study allow us to draw certain cognitive conclusions, illustrating the relationships between the studied variables. Due to the correlative research model and the small sample size, the conclusions are formulated in a mostly non-categorical manner.

1. Most probably, due to the nature of the discipline (the long duration of tennis matches, the great changeability of the situations and the multitude of stressors), tennis players use coping strategies more often than skiers, regardless of the nature of the strategy.

2. Using task-focused strategies more often, and resorting to emotion-focused and avoidance strategies less often, seems to help athletes to achieve a higher result during a competition. 
3. Studies on the factors which determine the effectiveness of coping strategies in sport should be broadened, and should encompass more numerous groups of sportspersons from various sport disciplines and at different skill levels.

Based on the obtained results, it is also possible to formulate certain practical tips for coaches and sportspersons:

1. Task-focused coping strategies should be developed in order to improve the competitive results of sportspersons.

2. Taking the situational nature of coping strategies into consideration, they can be designed and modified to improve the results.

\section{References}

1. Aldwin C.M. (2007) Stress, coping and development: An integrative perspective. Guilford Press, New York.

2. Allen M.S., D. Frings, S. Hunter (2012) Personality, coping, and challenge and threat states in athletes. Int. J. Sport Exerc. Psychol., 10: 264-275.

3. Anshel M.H., D.I. Anderson (2002) Coping with acute stress in sport: Linking athletes' coping style, coping strategies, affect, and motor performance. Anxiety, Stress, Coping, 15: 193-209.

4. Auweele Y.V., B. De Cuyper, V. Van Miele, R. Rzewnicki (1993) Elite performance and personality: From description and prediction to diagnosis and intervention. In: R.N. Singer, M. Murphy, L.K. Tennant (eds.) Handbook of research on sport psychology. MacMillan Publishing Company, New York, pp. 257-289.

5. Carver C.S., M.F. Scheier, J.K. Weintraub (1989) Assessing Coping strategies: a theoretically - based approach. J. Pers. Soc. Psychol., 56: 267-283.

6. Gardner F., Z.E. Moore (2006) Clinical sport psychology. Human Kinetics, Champaign.

7. Gaudreau P., J.P. Blondin (2002) Development of a questionnaire for the assessment of coping strategies employed by athletes in competitive sport settings. Psychol. Sport Exerc., 3: 1-34.

8. Gaudreau P., J.P. Blondin (2004) Different athletes cope differently during a sport competition: a cluster analysis of coping. Pers. Indiv. Differ., 36: 1865-1877.

9. Groppel J.L., S.F. Fiske (1996) How tennis relieves stress. Tennis, 31: 78.

10. Harwood C. (2009) Enhancing Self-Efficacy in Professional Tennis: Intensive Work for Life on the Tour. In:
B. Hemmings, T. Holder (eds.) Applied Sport Psychology: A Case-Based Approach. Wiley, New York, pp. 27-54.

11. Hemmings B., T. Holder (2009) Applied Sport Psychology: A Case-Based Approach. Wiley, New York.

12. Hurst J.R., A. Thompson, A.J. Visek, B. Fisher, P. Gaudreau (2011) Towards a dispositional version of the Coping Inventory for Competitive Sport. Int. J. Sport Psychol., 42: 167-185.

13. Kaiseler M., R.C.J. Polman, A.R. Nicholls (2009) Mental toughness, stress, stress appraisal, coping and coping effectiveness in sport. Pers. Indiv. Differ., 47: 728-733.

14. Loehr J.E. (1990) Providing sport psychology consulting services to professional tennis players. Sport Psychol, 4: 400-408.

15. Morris T., M. Spittle, A. Watt (2005) Imagery in sport: A complete picture. Human Kinetics, Champaign.

16. Murphy S. (2005) The Sport Psych handbook. Human Kinetics, Champaign.

17. Nicholls A.R., R.C.J. Polman, A.R. Levy (2012) A path analysis of stress appraisals, emotions, coping, and performance satisfaction among athletes. Psychol. Sport Exerc., 13: 263-270.

18. Peden A. (2007) Cognitive techniques to manager performance anxiety in tennis. ITF Coach. Sport Sci. Rev., 43: 12-13.

19. Pensgaard A.M., G.C. Roberts (2003) Achievement goal orientations and the use of coping strategies among Winter Olympians. Psychol. Sport Exerc., 4: 101-116.

20. Puente-Diaz R., M.H. Anshel (2005) Sources of acute stress, cognitive appraisal, and coping strategies among highly skilled Mexican and U.S. competitive tennis players. J. Soc. Psychol., 145: 429-446.

21. Vealey R.S. (2001) Understanding and enhancing selfconfidence in athletes. In: R.N. Singer, H.A. Hansenblas, C.M. Janelle (eds.) Handbook of sport psychology. Wiley, New York, pp. 550-565.

\section{Received 28.12.2015 \\ Accepted 15.03.2016}

\section{(C) University of Physical Education, Warsaw, Poland}

\section{Acknowledgments}

The study was conducted as part of the University of Physical Education young researchers project DM-34 entitled 'Factors determining the efficiency of coping with stress in tennis players and alpine skiers'. 